\title{
Artificial Neural Networks for Radiation Dose Prediction in Nuclear Emergencies
}

\author{
-Preliminary Investigations
}

\author{
Cláudio Márcio do Nascimento Abreu Pereira ${ }^{1, *}$, Roberto Schirru², Kelcio Gomes ${ }^{1}$ and José Luiz Cunha ${ }^{2}$ \\ ${ }^{1}$ Comissão Nacional de Energia Nuclear - IEN/CNEN.Rua Helio de Almeida, 75, Ilha do Fundão, 21941- 906 \\ Rio de Janeiro \\ ${ }^{2}$ Universidade Federal do Rio de Janeiro - PEN/COPPE/UFRJ. Ilha do Fundão, 21941-901, Centro de Tecnologia \\ Rio de Janeiro \\ ${ }^{*}$ Corresponding author
}

\begin{abstract}
This work investigates the use of Artificial Neural Networks (ANN) for radiation dose prediction due to a nuclear power plant (NPP) accident with radioactive material release. The main objective is to avoid necessity of using complex timeconsuming simulators during the emergency. Training, test and production data sets have been generated by realistic simulations on the precise atmospheric dispersion system used in CNAAA Brazilian NPP. Considering a hypothetical Lost of Coolant Accident (LOCA), several ANN architectures have been trained with a wide range of atmospheric conditions in order to predict spatial effective doses. As a result, a Backpropagation Multilayer Perceptron (MLP) with 5 layers demonstrated to achieve the best generalization, reaching a correlation factor of 0.990 for the validation dataset. On the other hand the GRNN reached a correlation factor slightly worse $(\mathbf{0 . 9 8 6 )}$ but very faster.
\end{abstract}

Keywords-artificial neural networks, radiation dose prediction, nuclear power plant, atmospheric dispersion

\section{INTRODUCTION}

An accurate prediction of atmospheric dispersion of radioactive material during a Nuclear Power Plant (NPP) emergency is very important to support decision related to people evacuation and environment protection. Such prediction generally involves simulation of complex physical models, such as: source term prediction, wind field calculations, plume dispersion, radionuclide deposition and equivalent doses prediction. These models are, however, much time consuming and need powerful computers to achieve the desired quality.

Aimed to overcome the need for such computational resources during emergencies, we investigate here the use of Artificial Neural Networks (ANN) [1] in the prediction of spatial doses.

The ANNs are trained offline, using data previously generated by the CNAAA Brazilian NPP atmospheric dispersion system (ADS) (simulator) considering a hypothetical Lost of Coolant Accident (LOCA) with its default releasing paths and NPP status. The ANNs were trained to predict spatial effective doses for a wide range of atmospheric conditions.

The main objective of this work is to evaluate the ability of ANNs in dealing with such complex task. To accomplish that, three ANN architectures have been investigated: two Backpropagation Multilayer Perceptrons (MLP) [1] and one General Regression Neural Networks (GRNN) [2].

The remaining of this paper is subdivided as follow. Section 2 contextualize and introduces the problem. Section 3 describes the ANN design and training. Results of the investigations are shown in section 4 and, finally, some concluding remarks are seen in section 5 .

\section{CONTEXTUALIZATION AND PROBLEM DESCRIPTION}

There are several works in literature that relates the use of ANN applied to radioactivity prediction.

Timonin and Savelieva [3] investigated the use of a GRNN for spatial predictions of radioactivity. They used the SIC2004 exercise as benchmark and concluded that GRNN is promising for spatial prediction of radioactivity.

Mól et al [4] also applied a GRNN for dose prediction inside the area of the Argonauta research reactor at Instituto de Engenharia Nuclear/CNEN (Brazil). In this work, dose prediction was done as function of reactor operating power and a given position.

Sarwat and Helal [5] used a GRNN for estimating workers internal dose. They emphasized that GRNNs are efficient for continuous functions mapping and concluded that they have good possibilities in the proposed application.

There are also several correlated works that do not deals with radiation, but is related to atmospheric dispersion.

Cao et al [6] applied ANN in prediction of short-term concentration distributions of aerosols released from point sources. He concluded that the performance of the neural network model was comparable or better than predictions from two Gaussian-based puff models.

Lauret et al [7], investigated the use of ANN in atmospheric gas dispersion concluding that the stationary ANN model gave good agreement with CFD software with the advantage of faster processing.

Hossain [8] applied ANN to predict concentration of carbon monoxide and particulate matters in urban atmospheres using 
field meteorological and traffic data. The conclusion was that ANN models based on both meteorological and traffic variables are capable of resolving patterns of pollutant dispersion to the atmosphere for different cities.

The present work is aimed to investigate the use of ANNs to predict doses due to atmospheric dispersion of radionuclide during a NPP emergency with radioactive material release. The computational experiments have been done considering the scenario in the neighborhood of CNAAA NPP site, at Angra dos Reis (Brazil), as illustrated in Figure 1.

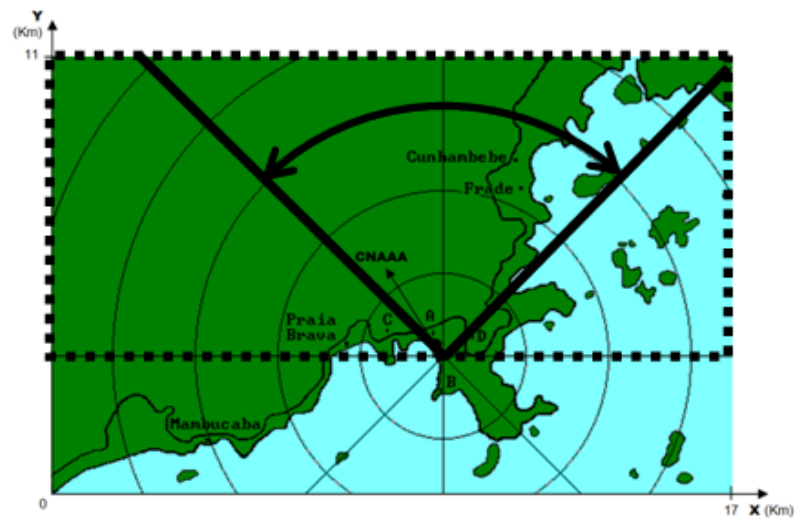

FIGURE I. REGION OF INTEREST FOR DOSE PREDICTION

The present work is aimed to investigate the use of ANNs to predict doses due to atmospheric dispersion of radionuclide during a NPP emergency with radioactive material release. The computational experiments have been done considering the scenario in the neighborhood of CNAAA NPP site, at Angra dos Reis (Brazil), as illustrated in Figure 1, considering that North is $0 \mathrm{o}$ and angles increase clockwise.

\section{NEURAL Networs Design AND Training}

Artificial Neural Networks (ANN) [1] are mathematical models inspired in human brain, which have the ability of learning by examples. There are many different approaches for ANNs. In this work, we consider those that use supervised learning (input/output learning) and are skilled for interpolation and prediction. According to literature, Backpropagation Multilayer Perceptrons (MLP) [1] and General Regression Neural Networks (GRNN) [2] present such characteristics.

MLPs are comprised by: i) an input layer, which receives the input data, ii) an output layer, which provides the ANN output and iii) one or more hidden layers. The number of neurons in the input layers is equal to the number of inputs of the problem. As well, the number of neurons in the output layer is equal to the number of outputs. The number of neurons in the hidden layer(s) is flexible and is responsible to provide the ability of non-linear adaptation of the ANN. The activation function of neurons may also be non-linear for complex adaptations. The training algorithm used is the backpropagation [1].

GRNN is "a memory-based network that provides estimates of continuous variables and converge to the underlying (linear or nonlinear) regression surface" [2]. It uses a one-pass learning algorithm that provides smooth transitions between training patterns even with sparse data in multidimensional spaces. GRNNs are based on the concepts of consistent estimators proposed by Parzen (1962). The estimated output (Equation 1) is a weighted average of all training patterns.

$$
\begin{gathered}
\hat{Y}(X)=\frac{\sum_{i=1}^{n} Y^{i} \exp \left(-\frac{D_{i}^{2}}{2 \sigma^{2}}\right)}{\sum_{i=1}^{n} \exp \left(-\frac{D_{i}^{2}}{2 \sigma^{2}}\right)} \\
D_{i}^{2}=\left(X-X^{i}\right)^{T}\left(X-X^{i}\right)
\end{gathered}
$$

Where $Y(X)$ is the estimated output; $X^{i}$ and $Y^{i}$ are the training patterns inputs and outputs, respectively; $X$ is an observed value to which an estimation is required; and $\sigma$ is the smoothing factor.

High values of $\sigma$ leads to more smooth function. To find optimum value for $\sigma$, optimization procedures can be used in order to minimize the least squared errors for a given test set.

\section{A. ANN Inputs and Outputs}

The ANNs are trained to predict doses based on position (X, Y), meteorological conditions, for a given postulated accident. Many meteorological conditions could be used as ANN inputs, such as: i) wind velocity; ii) wind direction; iii) wind stability; iv) temperature; v) temperature gradient; vi) rainfall index; among others. Similarly, several possible outputs could be used: i) equivalent doses; ii) effective doses; iii) dose rates etc. However, in this preliminary investigation a reduced set is considered. The ANN inputs/outputs used here are shown in Table 1 .

TABLE I. ANN INPUTS AND OUTPUTS

\begin{tabular}{|l|l|}
\hline \multirow{4}{*}{ Inputs } & Wind velocity \\
\cline { 2 - 2 } & Wind direction \\
\cline { 2 - 2 } & Position $\mathrm{X}$ \\
\cline { 2 - 2 } & Position $\mathrm{Y}$ \\
\hline Output & Dose rate \\
\hline
\end{tabular}

\section{B. Training, Test and Validation Patterns Generation}

This work used, as reference, the atmospheric dispersion system (ADS) used CNAAA NPP. This system is automatically started if an emergency occurs. In this case, monitored plant status as well as acquired meteorological data are used as inputs. The ADS might also be used in simulation mode, allowing realistic cases to be simulated using user defined inputs. Figure 2 illustrates the ADS used in this work to simulate training, test and validation data . 


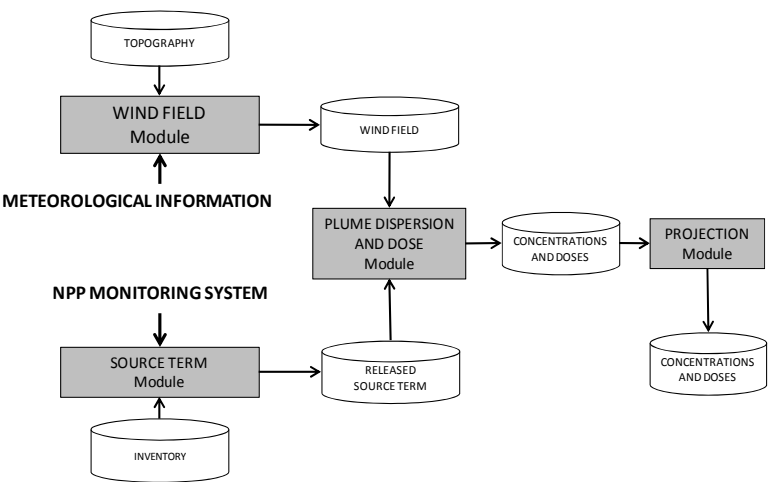

FIGURE II. SCHEMATIC DIAGRAM OF THE NPP ATMOSPHERIC DISPERSION SYSTEM

Using the ADS simulator shown in Figure 2, training, test and validation data have been generated, uniformly distributed on the space formed by the ANN input variables. Table 2 shows the ranges and distribution of each variable of training, test and production sets.

TABLE II. RANGES AND DISTRIBUTION OF TRAINING, TEST AND VALIDATION DATASETS

\begin{tabular}{|l|l|l|l|}
\hline Name & Range & Values & Step \\
\hline Wind velocity (m/s) & $1-5$ & 9 & 0.5 \\
\hline Wind direction (degrees) & $315-45$ & 9 & 11.25 \\
\hline Position X ${ }^{\text {a }}$ & $1-65^{*}$ & 17 & 4 \\
\hline Position Y ${ }^{\text {a }}$ & $10-42^{*}$ & 9 & 4 \\
\hline
\end{tabular}

Making all possible combinations of variables shown in Table 2, 12,393 patterns (input-output) have been generated. Through empirical optimization tests, a good usage distribution was defined, according to Table 3.

TABLE III. USAGE OF GENERATED PATTERNS

\begin{tabular}{|l|l|}
\hline Usage & Quantity \\
\hline Training & 3,825 \\
\hline Test & 2,448 \\
\hline Validation & 6,120 \\
\hline
\end{tabular}

Due to the complexity of the problem, a relatively great number of training patterns were needed. On the other hand, increasing the training set implies a great delay on training time. This cost/benefit relation was observed during preliminary tests

Note that Test set is also great in order to better evaluate stopping criteria on MLPs and better optimize smoothing factors on the GRNN. Validation data are not used during ANN training. They are only used to simulate the real use of the ANN.

In order to improve the ANNs efficiency in learning the training patterns, data have been normalized according to Equation 3.

$$
X_{N}=\frac{(X-\bar{X})}{S}
$$

where $X_{N}$ is the normalized value; $X$ is the original value; $\bar{X}$ is the average and $S$ is the standard deviation;

\section{The ANNs Architectures}

Two Backpropagation Multilayer Perceptrons (MLP) architectures and one GRNN have been investigated for the proposed application.

The simplest MLP (MLP-3Layers) was a 3-layer with logistic activation function in the hidden and output layers (input layer only scales and distributes the inputs). According to training data, input and output layers have 4 and 1 neurons, respectively. The hidden layer is comprised by 60 neurons.

The second MLP (MLP-5Layers) was a 5-layer with logistic activation function in hidden and output layers (input layer only scales and distributes the inputs). Input and output layers have 4 and 1 neurons, respectively. The 3 hidden layers are comprised by 20 neurons each.

The GRNN [2] architecture is a one-pass training and its architecture is casted by the training data (the hidden layer have one neuron for each training pattern). However, the smoothing factor is a critical parameter for good generalization. Hence, the GRNN used here was optimized by a Genetic Algorithm [9], aimed to find the best smoothing factor for the selected test set.

\section{RESUlts AND DisCUSSIONS}

In this section, numerical results obtained in design, training and application of proposed ANN are evaluated, compared and discussed. Tables 4 shows comparisons of the results obtained by each ANN architecture when applied to the validation dataset.

TABLE IV. STATISTICS OF MLP-1

\begin{tabular}{|l|l|l|l|l|}
\hline Architecture & Correlation & Máx.Err & Ave. Err & Trn. Time \\
\hline MLP-3Layers & 0.978 & 132.859 & 2.869 & $4: 59 \mathrm{~h}$ \\
\hline MLP-5Layers & 0.990 & 125.594 & 1.045 & $2: 37 \mathrm{~h}$ \\
\hline GRNN & 0.986 & 137.551 & 1.477 & $0: 23 \mathrm{~h}$ \\
\hline
\end{tabular}

Training/optimization times on Tables 4 are only for effect of relative comparisons. As training has been done on a Virtual Machine with emulated Windows XP 32 bits, such execution times do not reflect absolute performance that can be achieved on training times.

It could be observed that the best statistics were obtained for the MLP-5Layers. Errors and correlation for the validation dataset demonstrate very good generalization (ability to predict untrained patterns). On the other hand, the GRNN which presented slightly worse correlation and average error, was the fastest to be trained (about 6.8 times faster than the MLP5Layers).

MLP-3Layers was the worst one with 0.978 of correlation (not so bad), average error almost 3 times and training time about 2 times greater than MLP-5Layers.

Figures 3 and 4 illustrate graphically the influence of the numbers shown on Table 4 on dose predictions using the validation dataset. 


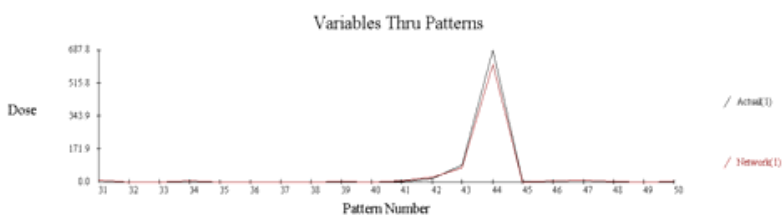

(a)

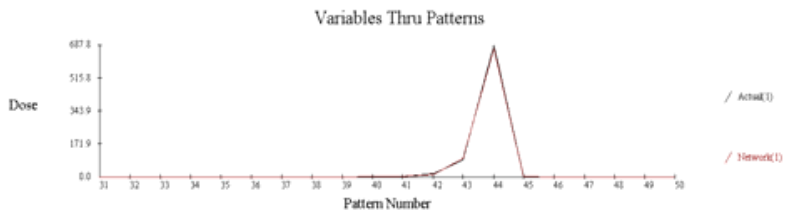

(b)

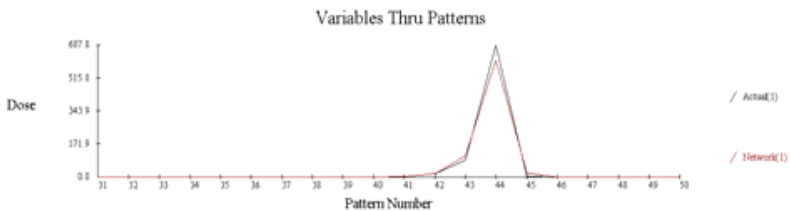

(c)

FIGURE III. FITTING ON HIGH OUTPUT (DOSE) VALUES. SIMULATED (ACTUAL) VERSUS PREDICTED (NETWORK) VALUES: (A) MLP-3LAYERS; (B) MLP-SLAYERS; (C) GRNN.

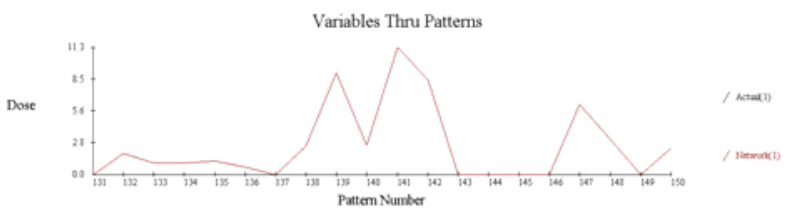

(a)

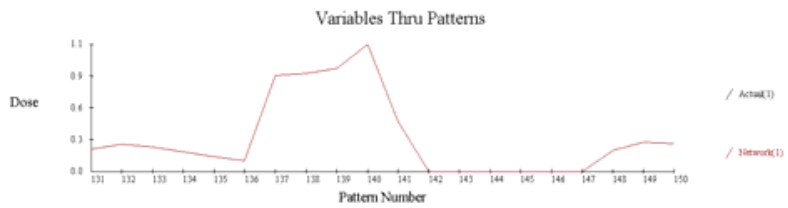

(b)

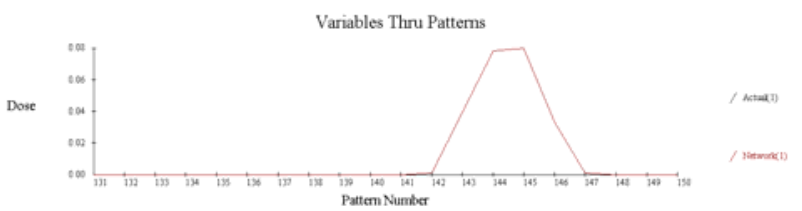

(c)

FIGURE IV. FITTING ON LOW OUTPUT (DOSE) VALUES. SIMULATED (ACTUAL) VERSUS PREDICTED (NETWORK) VALUES: (A) MLP-3LAYERS; (B) MLP-5LAYERS; (C) GRNN.

Note, in Figure 3, that for high values of output (dose), the fitting between simulated (label "actual(1)" in figures) and ANN prediction (label "network(1)" in figures) is good for all architectures, with certain advantage to MLP-5Layers.

However, for low values of output (dose), significant discrepancies may appear. Figure 4 shows a sub-set of the validation dataset which the simulated output should be zero. Observe that all ANN architectures present non null values. MLP-3Layers output reaches values about 11, while MLP5Layers got 1.1 and the GRNN found 0.08 .
Looking from this point of view, GRNN seems to be the best choice, however, looking deeper on the general fitting for high values of output, MLP-5Layers is slightly better.

\section{CONCLUSIONS}

In this work, 3 different architectures of ANN have been applied to radiation dose prediction in a realistic scenarios in Angra dos Reis, Brazil. The precise atmospheric dispersion system used in CNAAA NPP was used to simulate training patterns.

Results demonstrate that the Backpropagation Multilayer Perceptron with 5 layers (MLP-5Layers) is the better choice in terms of generalization, finding the best correlation coefficient (0.990) and the smaller average error.

Corroborating literature [5], GRNN also demonstrate to be efficient for continuous functions mapping. Although it presented a slightly worse correlation and average error, if has 2 advantages that could be considered: quick training (smoothing factor optimization) and better fit in small values.

\section{ACKNOWLEDGMENT}

Cláudio M.N. A. Pereira and Roberto Schirru are sponsored by Conselho Nacional de Desenvolvimento Científico e Tecnológico - CNPq.

\section{REFERENCES}

[1] Hayking, S. Neural Networks: A Comprehensive Foundation. Second Edition. Prentice Hall, 1999

[2] Specht, D. F. A General Regression Neural Network. IEEE Transactions on Neural Networks. vol.2, n.6, 1991

[3] Timonin, V. and Savelieva, E. Spatial Prediction of Radioactivity using General Regression Neural Networks. Applied GIS, vol.1, n.2, Monash University EPress, 2005

[4] Mól, A. C. A., Pereira, C. M. N. A. Freitas, V. G. G. and Jorge, C. A. F Radiation dose rate map interpolation in nuclear plants using neural networks and virtual reality techniques, Annals of Nuclear Energy, 38, pp.705-712, 2011

[5] Sarwat, E. and Helal, N. General Regression Neural Networks for Estimating Radiation Workers Internal Dose. Arab Journal of Nuclear Science and Applications, 46(1), (374-380), 2013.

[6] Cao, X., Roy, G., Hurley, W.J. and Andrews, W. S. Modeling the Concentration Distributions of Aerosol Puffs Using Artificial Neural Networks. Boundary-Layer Meteorology, 136(1):83-103, 2010 (DOI: 10.1007/s10546-010-9501-)

[7] Lauret, P., Heymesa, F., Aprina, L., Johannetb, A., Dusserrea, G. Munierc, L., Lapébiec, E.. Near Field Atmospheric Dispersion Modeling on an Industrial Site Using Neural Networks. Chemical Engineering Transactions, vol.31, 2013

[8] Hossain, K. M. A. Predictive Ability of Improved Neural Network Models to Simulate Pollutant Dispersion. International Journal of Atmospheric Sciences, 2014 (http://dx.doi.org/10.1155/2014/141923).

[9] Goldberg, D. E., Genetic Algorithms in Search, Optimization and Machine Learning, New York, Addison-Wesley, 1989. 